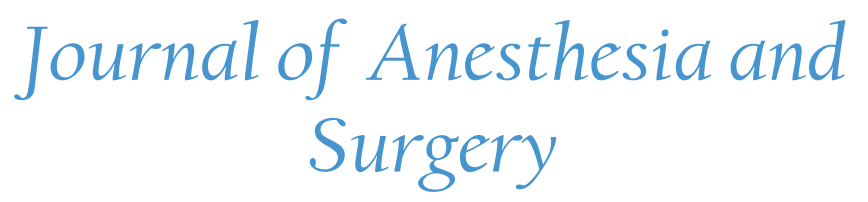

\title{
Adopting Information Management Based Joint Preoperative Assessment and Risk Stratification Model to Save Surgical Care Cost
}

\author{
Habib Md Reazaul Karim*
}

Assistant Professor, Department of Anaesthesiology, Andaman and Nicobar Island Institute of Medical Sciences \& GB Pant Hospital, Port Blair, India

*Corresponding author: Habib Md Reazaul Karim, Department of Anaesthesiology, ANIIMS \& GB Pant Hospital, Port Blair, India, Tel: +919612372585; E-mail: drhabibkarim@gmail.com

Citation: Karim, H.M.R. Adopting Information Management Based Joint Preoperative Assessment and Risk Stratification Model to Save Surgical Care Cost. (2017) J Anesth Surg 4(1): 7- 8.
Received date: December 21, 2016

Accepted date: January 20, 2017

Published date: January 25, 2017

DOI: $10.15436 / 2377-1364.17 .059$

\section{Introduction}

Preoperative assessment and risk stratification is an integral part of anesthetic care which is one of the various duties of an anesthesiologist. Laboratory investigation is an important element in the process of preoperative assessment and risk stratification. Unfortunately, despite of having negative recommendations for routine preoperative testing for more than a decade, it still remains a tradition in health care delivery for surgical patients ${ }^{[1,2]}$. The health care cost is becoming an increasing burden for every nation, especially for developing and underdeveloped countries. Studies conducted on cost savings have shown that $63 \%$ of the total cost of the tests was due to unnecessary tests ${ }^{[3,4]}$. Preoperative testing is estimated to cost US\$ 18 billion annually in United Sates alone ${ }^{[5]}$.

Efforts have been made by different health care societies and authorities to guide the preoperative testing before elective surgeries. American Society of Anaesthesiologists and the National Institute of Health and Clinical Excellence guidelines on preoperative testing are notable among them ${ }^{[1,6]}$. Unfortunately, the tradition of ordering routine preoperative tests is very much prevalent in clinical practice. Many of the tests ordered are actually unnecessary or unindicated, thus a good amount of cost saving is possible by avoiding such wrong traditional practice of routine testing ${ }^{[3,7-9]}$.

In preoperative risk assessment, the history and physical examination are the strongest predictors of perioperative complications. Ancillary tests should be indicated on an individual basis if the history and physical examination indicate towards some underlying disease ${ }^{[10]}$. However, studies show that the practice has not changed to 'individualized / patients char- acteristic' from 'routine' and that preoperative testing is more strongly associated with provider practice patterns than with patient characteristics ${ }^{[11]}$.

A study reviewing data from National Surgical Quality Improvement Program database found that neither laboratory testing nor abnormal results were associated with postoperative complications ${ }^{[12]}$. Many a time, we try to find some hidden abnormality by using routine preoperative testing. What we need to realize is that routine screening does not improve patient safety in the perioperative period. It is also necessary to differentiate between global screening method and preoperative risk evaluation. If the screening examination needs to be carried out, it should be independent of a planned surgery ${ }^{[13]}$. Although preoperative risk evaluation can, it should not fill this "gap" of screening examination ${ }^{[13]}$.

It has been found that most of the patients who attend preanesthesia clinic (PAC) for preoperative evaluation, risk assessment and stratification had already undergone majority of the tests on the very first contact with surgeon ${ }^{[9,14]}$. Studies also have shown that tests ordered by the anesthesiologists are more in compliance to the guidelines or recommendations than those ordered by the surgeons for preoperative evaluation ${ }^{[9,15]}$. So involvement of surgical discipline (by not ordering routine preoperative tests) is very much essential for reducing this unnecessary cost burden on patients, institution and nation. Anesthesiologists also need to be more rational and updated because even their practice is not up to the $\operatorname{mark}^{[8]}$. This is an era of information technology, and increasing adoption of information management systems in hospitals and health care enables anesthesiologists and surgeons to work hand in hand to save surgical care cost in preoperative assessment. Thus, information management 
based joint preoperative assessment and risk stratification model is proposed here.

1) Patient comes to contact in primary health centre (PHC) / emergency department (ED) / outpatient department (OPD) (history, physical examination and clinical findings, differential diagnosis (DD), bed side / point of care investigations intended for diagnosis only) $\rightarrow$ enter in patients' electronic health record file (PEHRF)

2) If it appears that there is need for surgery of surgical consultation $\rightarrow$ refer to surgeon (in case patient attended PHC or ED)

3) (Day 1) Surgeon examines patient, reviews PEHRF, shortens DD to provisional diagnosis and asks only diagnostic investigations, updates PEHRF with proposed surgery (provisional).

4) (Day 1 / same day) Anesthesiologist sitting in the PAC room or in the joint consultation room examines the patient, assess the functional status clinically by assessing metabolic equivalents of task (MET), reviews PEHRF and decides on individualized preoperative investigations to be done, orders it electronically and updates the PEHRF with risk class and advises for optimization if required.

5) (By same day or day 2) OPD patients attend laboratory or testing centre and after completing the procedure leaves for home. $\rightarrow$ Investigations required are done, updated in the PEHRF by the concerned persons.

6) (Day 2 or 3) Surgeon reviews the updated PEHRF, confirms the diagnosis and proposed surgery and forwards the electronic file to the Anesthesiologist in PAC. (Hospital may contact the patient if patient needs to be examined personally again).

7) (Day 2 or 3 ) Anaesthesiologist reviews the updated PEHRF, stratifies risk class, advices preoperative medication and others as required / asks for optimization before admission as required and updates the PEHRF.

8) Surgeon / Hospital give the date of admission for surgery based on available slot / protocol.

This proposed model was developed after observing the ED, OPD, PAC and surgical care delivery process in three government sector tertiary care hospitals of India. Referral from PHC was also taken in to account. However, variations are likely to exist in different health care set ups or corporate / private set ups; accordingly, this proposed model can be adapted as per their own hospital protocol.

To conclude, it is high time to abandon the practice of routine preoperative tests and adopt individualized investigations. Surgeons and anesthesiologists need to work together to get rid of the burden of unindicated / unnecessary investigations. Adopting an information management system based joint preoperative assessment is likely to help in this.

\section{Conflict of Interest: None}

Acknowledgement and Clarification: The author is thankful to Prof (Dr.) Ramesh MT, Head of the department of General Surgery of the same institute for reviewing the proposed model. The proposed model is the idea or concept of the author and it was developed after observing the ED, OPD, PAC and surgical care delivery process in three government sector tertiary care hospitals as well PHC delivery and referral services of those areas.

\section{References}

1) Committee on Standards and Practice Parameters., Apfelbaum, J.L., Connis, R.T., et al. Practice advisory for pre anesthesia evaluation: An updated report by the American Society of Anesthesiologists Task Force on Preanesthesia Evaluation. (2012) Anesthesiology 116(3): 522-538.

2) National Collaborating Centre for Acute Care (UK). Preoperative tests: The use of routine preoperative tests for elective surgery - Evidence, methods and guidance. (2003) London: National Institute of Clinical Excellence.

3) Keshavan, V.H., Swamy, C.M. Pre-operative laboratory testing: A prospective study on comparison and cost analysis. (2016) Indian J Anaesth 60(11): 838-842.

4) Ferrando, A., Ivaldi, C., Buttiglieri, A., et al. Guidelines for preoperative assessment: Impact on clinical practice and costs. (2005) Int J Qual Health Care 17(4): 323-329.

5) Balk, E.M., Earley, A., Hadar, N., et al. Benefits and Harms of Routine Preoperative Testing: Comparative Effectiveness. (2014) Rockville (MD): Agency for Healthcare Research and Quality (US).

6) National Guideline Centre (UK). Routine Preoperative Tests for Elective Surgery. (2016) London: National Institute for Health and Care Excellence (UK).

7) Garcia, A.P., Pastorio, K.A., Nunes, R.L., et al. Indication of preoperative tests according to clinical criteria: need for supervision. (2014) Braz J Anesthesiol 64(1): 54-61.

8) Ranasinghe, P., Perera, Y.S., Senaratne, J.A., et al. Preoperative testing in elective surgery: Is it really cost effective? (2011) Anesth Essays Res 5(1): 28-32.

9) Karim, H.M., Yunus, M., Bhattacharyya, P. An observational cohort study on pre-operative investigations and referrals: How far are we following recommendations? (2016) Indian J Anaesth 60(8): 552-559.

10) Böhmer, A.B., Wappler, F., Zwissler, B. Preoperative Risk Assessment-From Routine Tests to Individualized Investigation. (2014) Dtsch Arztebl Int 111(25): 437-446.

11) Chen, C.L., Lin, G.A., Bardach, N.S., et al. Preoperative Medical Testing in Medicare Patients Undergoing Cataract Surgery. (2015) N Engl J Med 372: 1530-1538.

12) Benarroch-Gampel, J., Sheffield, K.M., Duncan, C.B., et al. Preoperative Laboratory Testing in Patients Undergoing Elective, Low-Risk Ambulatory Surgery. (2012) Ann Surg 256(3): 518-528.

13) Wappler, F. In Reply: to Questionable Statement PMID: 25686387”. (2015) Dtsch Arztebl Int 112(5): 70.

14) Nikhar, S.A., Jayaram, K. Preoperative Investigations in ASA I and II Patients - Do We Really Follow Recommendations? (2016) Journal of Medical Science and Clinical Research 4: 13992-13998.

15) Finegan, B.A., Rashiq, S., McAlister, F.A., et al. Selective ordering of preoperative investigations by anaesthesiologists reduces the number and cost of tests. (2005) Can J Anesth 52: 575-580.
Ommega Online Publishers

Journal Title: Journal of Anesthesia and Surgery (JAS)

Journal Short Name: J Anesth Surg
Journal ISSN: 2377-1364

E-mail: anestheisa@ommegaonline.com

Website: www.ommegaonline.org 